\title{
ON DARBOUX INTEGRABILITY OF THE POLYNOMIAL DIFFERENTIAL SYSTEMS
}

\author{
Jaume Llibre and Xiang Zhang
}

\begin{abstract}
This is a survey on recent results on the Darboux integrability of polynomial vector fields in $\mathbb{R}^{n}$ or $\mathbb{C}^{n}$ with $n \geq 2$. We also provide an open question and some applications based on the existence of such first integrals.
\end{abstract}

\section{INTRODUCTION}

In many branches of applied mathematics, physics and, in general, in applied sciences appear nonlinear ordinary differential equations. If a differential equation or vector field defined on a real or complex manifold has a first integral, then its study can be reduced by one dimension. Therefore a natural question is: Given a vector field on a manifold, how to recognize if this vector field has a first integral defined on an open and dense subset of the manifold? In general this question has no a good answer up to now.

In this survey we provide sufficient conditions for the existence of a first integral for polynomial vector fields in $\mathbb{R}^{n}$ or $\mathbb{C}^{n}$ with $n \geq 2$. An open question which essentially goes back to Poincaré is presented. Finally some applications of the existence of this kind of first integrals to physical problems, centers, foci, limit cycles and invariant hyperplanes are mentioned.

\section{DARBouX ThEORY OF INTEGRABILITY}

Since any polynomial differential system in $\mathbb{R}^{n}$ can be thought as a polynomial differential system inside $\mathbb{C}^{n}$ we shall work only in $\mathbb{C}^{n}$. If our initial differential system is in $\mathbb{R}^{n}$, once we get a complex first integral of this system inside $\mathbb{C}^{n}$ the real and the imaginary parts of it are real first integrals. Moreover if that complex first integral is rational, the same occur for its real and imaginary parts. In short in the rest of the paper we shall work in $\mathbb{C}^{n}$.

In this section we study the existence of first integrals for polynomial vector fields in $\mathbb{C}^{n}$ through the Darboux theory of integrability. The algebraic theory of integrability is a classical one, which is related with the first part of the Hilbert's 16th problem [19]. This kind of integrability is usually called Darboux integrability, and it provides a link between the integrability of polynomial vector fields and the number of invariant algebraic hypersurfaces that they have (see Darboux [13] and Poincaré [36]). This theory shows the fascinating relationships between integrability (a topological phenomenon) and the existence of exact algebraic invariant hypersurfaces formed by solutions for the polynomial vector field. This theory is now known as the Darboux theory of integrability, see for more details the Chapter 8 of $[16]$.

2010 Mathematics Subject Classification. 34A34, 34C05, 34C14.

Key words and phrases. Polynomial differential systems, polynomial vector fields, Darboux integrability. 


\subsection{Polynomial vector fields in $\mathbb{C}^{n}$}

As usual $\mathbb{C}[x]=\mathbb{C}\left[x_{1}, \ldots, x_{n}\right]$ denotes the ring of all complex polynomials in the variables $x_{1}, \ldots, x_{n}$. We consider the polynomial vector field in $\mathbb{C}^{n}$

$$
\mathcal{X}=\sum_{i=1}^{n} P_{i}(x) \frac{\partial}{\partial x_{i}}, \quad x=\left(x_{1}, \ldots, x_{n}\right) \in \mathbb{C}^{n},
$$

where $P_{i}=P_{i}(x) \in \mathbb{C}[x]$ have no common factor for $i=1, \ldots, n$. The integer $d=\max \left\{\operatorname{deg} P_{1}, \ldots, \operatorname{deg} P_{n}\right\}$ is the degree of the vector field $\mathcal{X}$. Usually for simplicity the vector field $\mathcal{X}$ will be denoted by $\left(P_{1}, \ldots, P_{n}\right)$.

\subsection{Invariant algebraic hypersurfaces and Darboux polynomials}

Let $f=f(x) \in \mathbb{C}[x]$. We say that $\{f=0\} \subset \mathbb{C}^{n}$ is an invariant algebraic hypersurface or $f$ is a Darboux polynomial of the vector field $\mathcal{X}$ if there exists a polynomial $K_{f} \in \mathbb{C}[x]$ such that

$$
\mathcal{X}(f)=\sum_{i=1}^{n} P_{i} \frac{\partial f}{\partial x_{i}}=f K_{f}
$$

The polynomial $K_{f}$ is called the cofactor of $f$. Note that from this definition the degree of $K_{f}$ is at most $d-1$, and also that if a solution orbit of the vector field $\mathcal{X}$ has a point on $f=0$, then the whole orbit is contained in $f=0$. This justifies the name of invariant algebraic hypersurface, because it is invariant by the flow of the vector field $\mathcal{X}$.

Of course if the dimension $n$ is equal to 2 , then an invariant algebraic hypersurface is an invariant algebraic curve.

If the polynomial $f$ is irreducible in $\mathbb{C}[x]$, then we say that $f=0$ is an irreducible invariant algebraic hypersurface, or that $f$ is an irreducible Darboux polynomial.

We remark that in the definition of invariant algebraic hypersurface $f=0$ we always allow this curve to be complex; that is $f \in \mathbb{C}[x]$ even in the case of a real polynomial vector fields. As we will see this is due to the fact that sometimes for real polynomial vector fields the existence of a real first integral can be forced by the existence of complex invariant algebraic hypersurfaces, see for more details the chapter 8 of [16]. Of course when we look for a complex invariant algebraic hypersurface of a real polynomial system we are thinking of the real polynomial system as a complex one.

The next result shows that it is sufficient to look for the irreducible invariant algebraic hypersurfaces or irreducible Darboux polynomials.

Proposition 1. Suppose $f \in \mathbb{C}[x]$ and let $f=f_{1}^{n_{1}} \ldots f_{r}^{n_{r}}$ be its factorization into irreducible factors over $\mathbb{C}[x]$. Then for a polynomial vector field $\mathcal{X}, f=0$ is an invariant algebraic hypersurface with cofactor $K_{f}$ if and only if $f_{i}=0$ is an invariant algebraic hypersurface for each $i=1, \ldots, r$ with cofactor $K_{f_{i}}$. Moreover $K_{f}=n_{1} K_{f_{1}}+\ldots+n_{r} K_{f_{r}}$.

For a proof of Proposition 1 see for instance [22].

\subsection{Exponential factors}


If $f, g \in \mathbb{C}[x]$ are coprime, we write $(f, g)=1$. Suppose that $(f, g)=1$, we say that $\exp (g / f)$ is an exponential factor of the vector field $\mathcal{X}$ if there exists a polynomial $L_{e} \in \mathbb{C}[x]$ of degree at most $d-1$ such that

$$
\mathcal{X}(\exp (g / f))=\exp (g / f) L_{e} .
$$

The polynomial $L_{e}$ is called the cofactor of the exponential factor. It is easy to prove that if $\exp (g / f)$ is an exponential factor, then $f=0$ is an invariant algebraic hypersurface. For a proof see again see [22].

\subsection{Multiplicity of an invariant algebraic hypersurface or of a Darboux polynomial}

Let $\mathbb{C}_{m}[x]$ be the $\mathbb{C}$-vector space of polynomials in $\mathbb{C}[x]$ of degree at most $m$. Then it has dimension $R=\left(\begin{array}{c}n+m \\ n\end{array}\right)$. Let $v_{1}, \ldots, v_{R}$ be a basis of $\mathbb{C}_{m}[x]$. Denote by $M_{R}$ the $R \times R$ matrix

$$
\left(\begin{array}{cccc}
v_{1} & v_{2} & \ldots & v_{R} \\
\mathcal{X}\left(v_{1}\right) & \mathcal{X}\left(v_{2}\right) & \ldots & \mathcal{X}\left(v_{R}\right) \\
\vdots & \vdots & \ddots & \vdots \\
\mathcal{X}^{R-1}\left(v_{1}\right) & \mathcal{X}^{R-1}\left(v_{2}\right) & \ldots & \mathcal{X}^{R-1}\left(v_{R}\right)
\end{array}\right)
$$

where $\mathcal{X}^{k+1}\left(v_{i}\right)=\mathcal{X}\left(\mathcal{X}^{k}\left(v_{i}\right)\right)$. Then $\operatorname{det} M_{R}$ is called the $m$-th extactic polynomial of $\mathcal{X}$. From the properties of the determinant we note that the extactic polynomial is independent of the choice of the basis of $\mathbb{C}_{m}[x]$ up to a nonzero constant factor. Observe that if $f=0$ is an invariant algebraic hypersurface of degree $m$ of $\mathcal{X}$, then $f$ divides the polynomial $\operatorname{det} M_{R}$. This is due to the fact that if $f$ is a member of a basis of $\mathbb{C}_{m}[x]$, then $f$ divides the whole column in which $f$ is located.

We say that an irreducible invariant algebraic hypersurface $f=0$ of degree $m$ has defined algebraic multiplicity $k$ or simply algebraic multiplicity $k$ if $\operatorname{det} M_{R} \not \equiv 0$ and $k$ is the maximum positive integer such that $f^{k} \operatorname{divides} \operatorname{det} M_{R}$; and it has no defined algebraic multiplicity if $\operatorname{det} M_{R} \equiv 0$.

We remark that the matrix (1) already appears in the work of Lagutinskii (see also Dobrovol'skii et al [15]). For a modern definition of the $m$-th extactic hypersurface and a clear geometric explanation of its meaning, the readers can consult Pereira [34]. Christopher et al [11] assuming the irreducibility of the invariant algebraic curves used the extactic curve to study the algebraic multiplicity of invariant algebraic curves of planar polynomial vector fields, and prove the equivalence of the algebraic multiplicity with other three ones: the infinitesimal multiplicity, the integrable multiplicity and the geometric multiplicity.

\subsection{Multiplicity and exponential factors}

The next result presents a characterization under suitable assumptions of the algebraic multiplicity of an invariant algebraic hypersurface using the number of exponential factors of $\mathcal{X}$ associated with the invariant algebraic hypersurface. This characterization due to Llibre and Zhang [28] extends the algebraic multiplicity introduced by Christopher, Llibre and Pereira in [11] for invariant algebraic curves of $\mathbb{C}^{2}$ to invariant algebraic hypersurfaces of $\mathbb{C}^{n}$. This result is a key point in the Darboux theory of integrability in arbitrary dimension. 
Theorem 2. Let $\mathcal{X}$ be a polynomial vector field. For a given irreducible invariant algebraic hypersurface $f=0$ of $\mathcal{X}$ with $f$ of degree $m$, assume that $\mathcal{X}$ restricted to $f=0$ has no rational first integral. Then $f$ has a defined algebraic multiplicity $k$ if andonly if the vector field $\mathcal{X}$ has $k-1$ exponential factors $\exp \left(g_{i} / f^{i}\right)$, where $g_{i}$ is a polynomial of degree at most $i \cdot m$ and $\left(g_{i}, f\right)=1$, for $i=1, \ldots, k-1$.

We remark that if $\mathcal{X}$ is a planar vector field, then clearly Theorem 2 always holds without the assumption on the non-existence of rational first integrals on $f=0$. For higher dimensional systems the assumption is necessary as the following example shows.

The real polynomial differential system

$$
\dot{x}=1, \quad \dot{y}=y(y-2 z), \quad \dot{z}=-z(y-z),
$$

has $z=0$ as an invariant plane of multiplicity 2 and its restriction to $z=0$ has the rational first integral $x+1 / y$. But this system has no exponential factor associated with $z=0$ as it is proved in the appendix.

This example shows that the additional assumption on the non-existence of the rational first integral on the invariant algebraic hypersurface for polynomial vector fields of dimension larger than 2 is necessary.

\subsection{Darboux first integral}

Let $M$ be an open and dense subset of $\mathbb{C}^{n}$. A non-constant function $H: M \rightarrow \mathbb{C}$ is a first integral of the polynomial vector field $\mathcal{X}$ on $M$ if it is constant on all orbits of $\mathcal{X}$ contained in $M$; i.e. $H(x(t))=$ constant for all values of $t$ for which the solution $x(t)$ is defined and contained in $M$. Clearly $H$ is a first integral of $\mathcal{X}$ on $M$ if and only if $\mathcal{X}(H)=0$ on $M$. Of course a rational first integral is a first integral given by a rational function, defined in the open subset of $\mathbb{C}^{n}$ where its denominator does not vanish. A Darboux first integral is a first integral of the form

$$
\left(\prod_{i=1}^{r} f_{i}^{l_{i}}\right) \exp (g / h),
$$

where $f_{i}, g$ and $h$ are polynomials, and the $l_{i}$ 's are complex numbers.

\subsection{Classical Darboux theory of integrability in $\mathbb{C}^{n}$}

The classical Darboux theory of integrability in $\mathbb{C}^{n}$ with $n \geq 2$ is summarized in the next theorem.

Theorem 3. Assume that the polynomial vector field $\mathcal{X}$ in $\mathbb{C}^{n}$ of degree $d>0$ has irreducible invariant algebraic hypersurfaces $f_{i}=0$ for $i=1, \ldots, p$ such that $f_{i}$ are pairwise relatively prime. Then the following statements hold.

(a) If $p \geq N+1$, then the vector field $\mathcal{X}$ has a Darboux first integral, where $N=\left(\begin{array}{c}n+d-1 \\ n\end{array}\right)$.

(b) If $p \geq N+n$, then the vector field $\mathcal{X}$ has a rational first integral.

Statement $(a)$ of Theorem 3 is due to Darboux $[13,14]$, and statement $(b)$ of Theorem 3 was proved by Jouanolou [20]. For a short proof of statement $(b)$ see Christopher and Llibre [8,9] for $n=2$ and Llibre and Zhang [30] for $n \geq 2$.

\subsection{Darboux theory of integrability in $\mathbb{C}^{n}$}


The following theorem improves the classical Darboux theory of integrability taking into account not only the invariant algebraic hypersurfaces but also their algebraic multiplicities.

Theorem 4. Assume that the polynomial vector field $\mathcal{X}$ in $\mathbb{C}^{n}$ of degree $d>0$ has irreducible invariant algebraic hypersurfaces $f_{i}=0$ such that the $f_{i}$ are pairwise relatively prime.

(i) If one of these irreducible invariant algebraic hypersurfaces has no defined algebraic multiplicity, then the vector field $\mathcal{X}$ has a rational first integral.

(ii) Suppose that all the irreducible invariant algebraic hypersurfaces $f_{i}=0$ have defined algebraic multiplicity $q_{i}$ for $i=1, \ldots, p$. If $\mathcal{X}$ restricted to each hypersurface $f_{i}=0$ with multiplicity larger than 1 has no rational first integral, then the following statements hold.

(a) If $\sum_{i=1}^{p} q_{i} \geq N+1$, then the vector field $\mathcal{X}$ has a Darboux first integral, where $N$ is the number defined in Theorem 3.

(b) If $\sum_{i=1}^{p} q_{i} \geq N+n$, then the vector field $\mathcal{X}$ has a rational first integral.

Statement $(i)$ follows from the second part of Theorem 3 of Pereira [34] (see also Theorem 5.3 of [11] for dimension 2). Statement (ii) for dimension 2 (i.e $n=2$ ) follows from [11] and for $n>2$ it is proved in [28].

Under the assumption $(b)$ of Theorem 4 any orbit of the vector field $\mathcal{X}$ is contained in an invariant algebraic hypersurface. We remark that if the vector field $\mathcal{X}$ is 2-dimensional, then the assumption on the non-existence of rational first integral of $\mathcal{X}$ restricted to the invariant algebraic curves is not necessary.

\subsection{Darboux theory of integrability in $\mathbb{R}^{n}$ taking into account the multi- plicity of the hyperplane at infinity}

In this subsection we show that if the hyperplane at infinity has multiplicity greater than 1 , then we can go further improving the Darboux theory of integrability taking into account the multiplicity of the hyperplane at infinity.

In order to use the infinity of $\mathbb{R}^{n}$ as an additional invariant hyperplane for studying the integrability of the vector field $\mathcal{X}$, we need the Poincaré compactification for the vector field $\mathcal{X}$, see for more details Cima and Llibre [12]. In the chart $U_{1}$ using the change of variables

$$
x_{1}=\frac{1}{z}, \quad x_{2}=\frac{y_{2}}{z}, \quad \ldots, \quad x_{n}=\frac{y_{n}}{z} .
$$

the vector field $\mathcal{X}$ is transformed to

$$
\overline{\mathcal{X}}=-z \bar{P}_{1}(y) \frac{\partial}{\partial z}+\left(\bar{P}_{2}(y)-y_{2} \bar{P}_{1}(y)\right) \frac{\partial}{\partial y_{2}}+\ldots+\left(\bar{P}_{n}(y)-y_{n} \bar{P}_{1}(y)\right) \frac{\partial}{\partial y_{n}},
$$

where $\bar{P}_{i}=z^{d} P_{i}\left(1 / z, y_{2} / z, \ldots, y_{n} / z\right)$ for $i=1, \ldots, n$ and $y=\left(z, y_{2}, \ldots, y_{n}\right)$. We note that $z=0$ is an invariant hyperplane of the vector field $\overline{\mathcal{X}}$ and that the infinity of $\mathbb{R}^{n}$ may be identified with $z=0$ of the vector field $\overline{\mathcal{X}}$. So we can define the algebraic multiplicity of $z=0$ for the vector field $\overline{\mathcal{X}}$.

We say that the infinity of $\mathcal{X}$ has defined algebraic multiplicity $k$ or simply algebraic multiplicity $k$ if $z=0$ has defined algebraic multiplicity $k$ for the vector field 
$\overline{\mathcal{X}}$; and that it has no defined algebraic multiplicity if $z=0$ has no defined algebraic multiplicity for $\overline{\mathcal{X}}$

In [39] Schlomiuk and Vulpe gave a definition for the algebraic multiplicity of the line at infinity for a planar vector field using a limit inside the definition. In fact the two definitions are equivalent. But ours is easier to apply for computing the algebraic multiplicity of the line at infinity for a given planar vector field.

Similar to Theorem 2 we have the following result characterizing the existence of exponential factors associated with the hyperplane at infinity.

Theorem 5. Assume that $\overline{\mathcal{X}}$ restricted to $z=0$ has no rational first integral. Then $z=0$ has algebraic multiplicity $k$ for $\overline{\mathcal{X}}$ if and only if $\overline{\mathcal{X}}$ has $k-1$ exponential factors $\exp \left(\bar{g}_{i} / z^{i}\right), i=1, \ldots, k-1$ with $\bar{g}_{i} \in \mathbb{C}_{i}[y]$ having no factor $z$.

The following result improves the Darboux theory of integrability in $\mathbb{R}^{n}$ taking into account the algebraic multiplicity of the hyperplane at infinity, for a proof see [29].

Theorem 6. Assume that the polynomial vector field $\mathcal{X}$ in $\mathbb{R}^{n}$ of degree $d>0$ has irreducible invariant algebraic hypersurfaces $f_{i}=0$ for $i=1, \ldots, p$ such that the $f_{i}$ are pairwise relatively prime.

(i) If one of these irreducible invariant algebraic hypersurfaces or the invariant hyperplane at infinity has no defined algebraic multiplicity, then the vector field $\mathcal{X}$ has a rational first integral.

(ii) Suppose that all the irreducible invariant algebraic hypersurfaces $f_{i}=0$ have defined algebraic multiplicity $q_{i}$ for $i=1, \ldots, p$ and that the invariant hyperplane at infinity has defined algebraic multiplicity $k$. If the vector field restricted to each invariant hypersurface including the hyperplane at infinity having algebraic multiplicity larger than 1 has no rational first integral, then the following hold.

(a) If $\sum_{i=1}^{p} q_{i}+k \geq N+2$, then the vector field $\mathcal{X}$ has a Darboux first integral, where $N=\left(\begin{array}{c}n+d-1 \\ n\end{array}\right)$.

(b) If $\sum_{i=1}^{p} q_{i}+k \geq N+n+1$, then the vector field $\mathcal{X}$ has a rational first integral.

We note that if the hyperplane at infinity is not taken into account, then Theorem 6 is exactly Theorem 4 . Also if the hyperplane at infinity has algebraic multiplicity 1 , then it does not contribute to integrability by comparing Theorem 6 with Theorem 4.

We remark that for the moment we do not have an analogous to Theorem 6 for polynomial vector fields in $\mathbb{C}^{n}$ which takes into account the multiplicity of the infinity. In [29] are shown some difficulties for obtaining the extension of Theorem 6 from polynomial vector fields in $\mathbb{R}^{n}$ to polynomial vector fields in $\mathbb{C}^{n}$.

In the previous subsection we showed by an example that the assumption on the non-existence of rational first integral of $\mathcal{X}$ restricted to an invariant algebraic hypersurface with multiplicity larger than 1 is necessary for the vector field in $\mathbb{R}^{n}$ with $n>2$. There are also examples showing that if $n>2$, the additional assumption is also necessary for the hyperplane at infinity with multiplicity larger 
than 1 , see [29]. If $\mathcal{X}$ is a planar vector field, then this additional assumption about the rational first integral is not necessary, it would imply that such a curve consists of singular points only. We have the following

Corollary 7. Assume that the polynomial vector field $\mathcal{X}$ in $\mathbb{R}^{2}$ of degree $d>0$ has irreducible invariant algebraic curves $f_{i}=0$ with defined algebraic multiplicity $q_{i}$ for $i=1, \ldots, p$ and that the invariant straight line at infinity has defined algebraic multiplicity $k$. Then the following hold.

(a) If $\sum_{i=1}^{p} q_{i}+k \geq\left(\begin{array}{c}d+1 \\ 2\end{array}\right)+2$, then the vector field $\mathcal{X}$ has a Darboux first integral.

(b) If $\sum_{i=1}^{p} q_{i}+k \geq\left(\begin{array}{c}d+1 \\ 2\end{array}\right)+3$, then the vector field $\mathcal{X}$ has a rational first integral.

\subsection{Construction of the first integrals}

From the previous main results of this section it follows easily statements (ii) and (iii) of the next theorem. Statements (i) and (iv) without taking into account the cofactors of the exponential factors are essentially due to Darboux [13]. The rest of the statements come from Christopher and Llibre [8, 9].

Theorem 8. Suppose that a polynomial vector field $\mathcal{X}$ of degree $d$ in $\mathbb{C}^{n}$ admits $p$ irreducible invariant algebraic hypersurfaces $f_{i}=0$ such that the $f_{i}$ are pairwise relatively prime with cofactors $K_{i}$ for $i=1, \ldots, p$ and $q$ exponential factors $\exp \left(g_{j} / h_{j}\right)$ with cofactors $L_{j}$ for $j=1, \ldots, q$.

(i) There exist $\lambda_{i}, \mu_{j} \in \mathbb{C}$ not all zero such that $\sum_{i=1}^{p} \lambda_{i} K_{i}+\sum_{j=1}^{q} \mu_{j} L_{j}=0$, if and only if the (multi-valued) function

$$
f_{1}^{\lambda_{1}} \ldots f_{p}^{\lambda_{p}}\left(\exp \left(\frac{g_{1}}{h_{1}}\right)\right)^{\mu_{1}} \ldots\left(\exp \left(\frac{g_{q}}{h_{q}}\right)\right)^{\mu_{q}}
$$

is a first integral of $\mathcal{X}$.

(ii) If $p+q \geq N+1$, then there exist $\lambda_{i}, \mu_{j} \in \mathbb{C}$ not all zero such that $\sum_{i=1}^{p} \lambda_{i} K_{i}+$ $\sum_{j=1}^{q} \mu_{j} L_{j}=0$

(iii) If $p+q \geq N+n$, then $\mathcal{X}$ has a rational first integral.

In the particular case that $n=2$ the following statements also hold.

(iv) There exist $\lambda_{i}, \mu_{j} \in \mathbb{C}$ not all zero such that $\sum_{i=1}^{p} \lambda_{i} K_{i}+\sum_{j=1}^{q} \mu_{j} L_{j}=-\operatorname{div}(P, Q)$, if and only if function (3) is an integrating factor of $\mathcal{X}$.

(v) If $p+q=N$ then function (3) is a first integral if $\sum_{i=1}^{p} \lambda_{i} K_{i}+\sum_{j=1}^{q} \mu_{j} L_{j}=0$, or an integrating factor if $\sum_{i=1}^{p} \lambda_{i} K_{i}+\sum_{j=1}^{q} \mu_{j} L_{j}=-\operatorname{div}(P, Q)$, under the condition that not all $\lambda_{i}, \mu_{j} \in \mathbb{C}$ are zero. 


\section{First integrals obtained By the DARBoux THEORY OF INTEGRABiLity IN DIMENSION TWO}

In this section we summarize the effectiveness of the Darboux theory of integrability in dimension two, i.e. which sort of integrals does it capture. For additional details on this section see [16], Prelle and Singer [37], and Singer [40].

The idea of determining what type of functions can arise as the result of evaluating an indefinite integral or solving a differential equation goes back to Liouville. The modern formulation of these ideas is usually done through differential algebra. Some of the advantages over an analytic approach are first that the messy details of branch points etc, are hidden completely, and second the Darboux theory of integrability can be studied using symbolic computation.

\subsection{Elementary and Liouvillian functions}

We assume that the set of functions we are interested in forms a field together with a number of derivations. We call such an object a differential field. The process of adjoining more functions to a given set of functions is described by a tower of such fields:

$$
F_{0} \subset F_{1} \subset \cdots \subset F_{n} .
$$

Of course, we must also specify how the derivations of $F_{0}$ are extended to derivations on each $F_{i}$.

The fields we are interested in arise by adding exponentials, logarithms or the solutions of algebraic equations based on the previous set of functions. That is we take

where one of the following holds:

$$
F_{i}=F_{0}\left(\theta_{1}, \ldots \theta_{i}\right)
$$

(i) $\delta \theta_{i}=\theta_{i} \delta g$, for some $g \in F_{i-1}$ and for each derivation $\delta$;

(ii) $\delta \theta_{i}=g^{-1} \delta g$, for some $g \in F_{i-1}$ and for each derivation $\delta$;

(iii) $\theta_{i}$ is algebraic over $F_{i-1}$.

If we have such a tower of fields, $F_{n}$ is called an elementary extension of $F_{0}$.

This is essentially what we mean by a function being expressible in closed form. We call the set of all elements of a differential field which are annihilated by all the derivations the field of constants. We shall always assume that the field of constants is algebraically closed.

We say that our system has an elementary first integral if there is an element $u$ in an elementary extension field of the field of rational functions $\mathbb{C}(x, y)$ with the same field of constants such that $D u=0$. The derivations on $\mathbb{C}(x, y)$ are of course $d / d x$ and $d / d y$.

Another class of integrals we are interested in are the Liouvillian ones. Here we say that an extension $F_{n}$ is a Liouvillian extension of $F_{0}$ if there is a tower of differential fields as above which satisfies conditions (i), (iii) and

$(i i)^{\prime} \delta_{\alpha} \theta_{i}=h_{\alpha}$ for some elements $h_{\alpha} \in F_{i-1}$ such that $\delta_{\alpha} h_{\beta}=\delta_{\beta} h_{\alpha}$.

This last condition, mimics the introduction of line integrals into the class of functions. Clearly $(i i)$ is included in $(i i)^{\prime}$.

This class of functions represents those functions which are obtainable "by quadratures". An element $u$ of a Liouvillian extension field of $\mathbb{C}(x, y)$ with the same field of constants is said to be a Liouvillian first integral. 
A function of the form $e^{w_{0}+\sum c_{i} \ln \left(w_{i}\right)}$, where $c_{i}$ are constants and $w_{i}$ are rational functions is called Darboux function.

As we shall see the Darboux theory of integrability finds all Liouvillian first integrals of the planar polynomial vector fields.

\subsection{The relation between a first integral and its associated integrating factor}

We consider the following classes of functions, polynomial, rational, Darboux, elementary and Liouvillian. We note that each of these classes of functions is contained in the following one of the previous list. The simplest functions which can be first integrals of polynomial differential systems are functions of one of the mentioned classes. The Darboux theory of integrability allows to compute all the first integrals belonging to one of these classes, see $[16,37,40]$. In the next result and for polynomial differential systems in $\mathbb{R}^{2}$ or $\mathbb{C}^{2}$ we summarize the explicit relationships between the functions defining the first integrals and their integrating factors.

Theorem 9. Let $\mathcal{X}$ be a planar polynomial vector field whose components are relatively prime.

(a) If $\mathcal{X}$ has a Liouvillian first integral, then it has a Darboux integrating factor.

(b) If $\mathcal{X}$ has an elementary first integral, then it has an integrating factor of the form a rational function to power $1 / n$ for positive integer $n$.

(c) If $\mathcal{X}$ has a Darboux first integral, then it has a rational integrating factor.

(d) If $\mathcal{X}$ has a rational first integral, then it has a rational integrating factor.

(e) If $\mathcal{X}$ has a polynomial first integral, then it has a polynomial integrating factor

Statement (a) is due to Singer [40], see also Christopher [7] and Pereira [35]. Statement (b) was proved by Prelle and Singer [37]. Statement (c) was shown by Chavarriga, Giacomini, Giné and Llibre in [4]. The proof of statement (d) follows easily. Finally the proof of statement (e) follows from [4] and [17], this last paper is due to Ferragut, Llibre and Mahdi.

\section{AN OPEN QUESTION FOR PLANAR POLYNOMIAL VECTOR FIELDS}

In all this section $n=2$.

From Jouanolou's result (see Theorem 4 (ii.b)) it follows that for a given planar polynomial differential system of degree $d$ the maximum degree of its irreducible invariant algebraic curves is bounded, since either it has a finite number $p<[d(d+$ $1) / 2]+2$ of invariant algebraic curves, or all its trajectories are contained in invariant algebraic curves and the system admits a rational first integral. Thus for each polynomial differential system there is a natural number $N$ which bounds the degree of all its irreducible invariant algebraic curves. A natural question which goes back to Poincaré [36] is: to give an effective procedure to find $N$. Partial answers to this question were given by Cerveau and Lins Neto [3], Carnicer [2], Campillo and Carnicer [1], and Walcher [42]. These results depend on either restricting the nature of the polynomial differential system, or more specifically on the singularities of its invariant algebraic curves.

Of course, given such a bound for $N$, it is then easy to compute the invariant algebraic curves of the system and also describe its elementary or Liouvillian first 
integrals (modulo any exponential factors) see for instance Man and Maccallum[31], Christopher [6],and Pearson, Lloyd and Christopher[33].

Unfortunately for the class of polynomial differential systems with fixed degree $d$, there does not exist a uniform upper bound $N(d)$ for $N$ as shown by the polynomial differential system of degree 1 :

$$
\dot{x}=r x, \quad \dot{y}=s y,
$$

with $r$ and $s$ be positive integers. This system has a rational first integral

$$
H=\frac{y^{r}}{x^{s}} \text {. }
$$

and hence invariant algebraic curves $x^{s}-h y^{r}=0$ for all $h \in \mathbb{C} \backslash\{0\}$ and $r$ and $s$ relatively prime.

A conjecture (coming from Poincaré) was that the following question would have a positive answer:

For a given $d \geq 2$ is there a positive integer $M(d)$ such that if a polynomial vector field of degree $d$ has an irreducible invariant algebraic curve of degree $\geq M(d)$, then it has a rational first integral.

See for instance the open question 2 of Christopher and Llibre [9], or the question at the end of the introduction of Lins Neto [21].

The conjecture has a negative answer, two counterexamples appeared at the same time, one due to Moulin Ollagnier [32], and another due to Christopher and Llibre [10]. Later on other counterexamples appeared see for instance Chavarriga and Grau [5]. But all these counterexamples exhibit a Darboux first integral or a Darboux integrating factor. So we conjecture that the following open question would have a positive answer(see [22]):

There is some number $D(d)$ for which any polynomial differential system of degree d having some irreducible invariant algebraic curve of degree $\geq D(d)$ has a Darboux first integral or Darboux integrating factor.

\section{Some APPlications}

The Darboux theory of integrability has been successfully applied to the study of some physical models. Thus, for instance, for the classical Bianchi IX system, and for the Einstein-Yang-Mills differential equations, Llibre and Valls in [25] and [26] provided a complete description of its Darboux polynomials, exponential factors, rational first integrals and Darboux first integrals. Similar studies was done by Valls [41] for the Rikitake system, and for the Lorenz system by Llibre and Zhang [27] and Zhang [43].

The proof of the classification of all centers of planar polynomial differential systems of degree 2 can be strongly simplified using the Darboux first integrals, see Schlomiuk [38] and the chapter 8 of Dumortier, Llibre and Artés [16].

Darboux first integrals can be used for obtaining new classes of integrable planar polynomial differential systems having a focus, see Giné and Llibre [18].

Using the Darboux theory of integrability Llibre and Rodríguez in [24] proved that every finite configuration of disjoint simple closed curves of the plane is topologically realizable as the set of limit cycles of a polynomial vector field. Moreover the realization can be made by algebraic limit cycles, and explicit polynomial vector fields exhibiting any given finite configuration of limit cycles are given. 
In [23] Llibre and Medrado gave best possible upper bounds of the maximal number of invariant hyperplanes, of the maximal number of parallel invariant hyperplanes, and of the maximal number of invariant hyperplanes that pass through a single point for the polynomial vector fields in $\mathbb{C}^{n}$ with a given degree.

\section{Appendix}

Proposition 10. The real polynomial differential system

$$
\dot{x}=1, \quad \dot{y}=y(y-2 z), \quad \dot{z}=-z(y-z),
$$

has no exponential factors associated to the invariant plane $z=0$ of multiplicity 2 .

Proof. By contrary we assume that system (4) has an exponential factor associated with $z=0$. Let $E=\exp \left(g / z^{s}\right)$ be the exponential factor with cofactor $L$, where $g$ is a polynomial of degree at most $s \geq 1$, and $L$ is a polynomial of degree at most 1 .

By the definition of exponential factor we get that $g$ should satisfy the equation

$$
\frac{\partial g}{\partial x}+y(y-2 z) \frac{\partial g}{\partial y}-z(y-z) \frac{\partial g}{\partial z}=-s(y-z) g+L z^{s} .
$$

Set $L=L_{0}+L_{1}$, where $L_{0}$ is a constant and $L_{1}$ is a homogeneous polynomial of degree 1 . In what follows we also denote by $g_{i}$ the homogeneous polynomial of degree $i$ of $g$.

If $\operatorname{deg} g=s$, equating the homogeneous part of degree $s+1$ of (5) we get

$$
y(y-2 z) \frac{\partial g_{s}}{\partial y}-z(y-z) \frac{\partial g_{s}}{\partial z}=-s(y-z) g_{s}+L_{1} z^{s}
$$

Using the Euler's formula for homogeneous functions this last equation is equivalent to

$$
x(y-z) \frac{\partial g_{s}}{\partial x}+y(2 y-3 z) \frac{\partial g_{s}}{\partial y}=L_{1} z^{s} .
$$

We claim that $L_{1} \equiv 0$. For proving it, we set $L_{1}=a x+b y+c z$ and write equation (6) in

$$
x(y-z) \frac{\partial g_{s}}{\partial x}=(a x+b y+c z) z^{s}-y(2 y-3 z) \frac{\partial g_{s}}{\partial y} .
$$

Clearly we must have $c=0$. Also we must have $b=0$, because $x$ divides $b y z^{s}-$ $y(2 y-3 z) \frac{\partial g_{s}}{\partial y}$. Finally we prove $a=0$. Since $x$ divides $\partial g_{s} / \partial x$, we set $\partial g_{s} / \partial x=$ $x h_{s-2}(x, y, z)$ with $h_{s-2}$ a homogeneous polynomial of degree $s-2$. It follows from (7) that

$$
(y-z) \frac{\partial g_{s}}{\partial x}=a z^{s}-y(2 y-3 z) h_{s-2}(x, y, z) .
$$

If $a \neq 0$, we must have $\frac{\partial g_{s}}{\partial x}=-a z^{s-1}$. But from (8) we are in contradiction. Hence we must have $a=0$, and consequently $L_{1}(x, y, z) \equiv 0$. The claim follows.

Now we will prove that $g_{s}=r z^{s}$. Set

$$
g_{s}(x, y, z)=z g_{s-1}(x, y, z)+q_{s}(x, y)
$$

with $g_{s-1}$ and $q_{s}$ homogenous polynomials of degrees $s-1$ and $s$, respectively. Substituting the expression of $g_{s}$ into (6) and set $z=0$, we get

$$
x \frac{\partial q_{s}}{\partial x}+2 y \frac{\partial q_{s}}{\partial y}=0 .
$$


This last equation can be written in

$$
y \frac{\partial q_{s}(x, y)}{\partial y}+s q_{s}(x, y)=0
$$

Its general solution is $q_{s}(x, y)=c(x) y^{-s}$. So we must have $q_{s}(x, y) \equiv 0$. Now set

$$
g_{s-1}(x, y, z)=z g_{s-2}(x, y, z)+q_{s-1}(x, y),
$$

with $g_{s-2}$ and $q_{s-1}$ homogenous polynomials of degrees $s-2$ and $s-1$, respectively. Then similarly we can prove from $(6)$ that $q_{s-1}(x, y) \equiv 0$, and so

$$
g_{s}(x, y, z)=z^{2} g_{s-2}(x, y, z) \text {. }
$$

By induction we can prove that $g_{s}=r z^{s}$.

The above proof shows that the exponential factor $E=\exp \left(g / z^{s}\right)$ (if exists) has a constant cofactor and $g=h(x, y, z)+r z^{s}$ with $h$ a polynomial of degree at most $s-1$. But in this case the exponential factor $E$ is essentially the same with $g$ of degree less than $s$.

If $\operatorname{deg} g=s-1$, equating the homogeneous part of degree $s$ of equation (5) we get that $L=L_{0}$ and $g_{s-1}$ satisfies the following equation

$$
y(y-2 z) \frac{\partial g_{s-1}}{\partial y}-z(y-z) \frac{\partial g_{s-1}}{\partial z}=-s(y-z) g_{s-1}+L_{0} z^{s} .
$$

If $s=1$, it is easy to show that $g_{s-1}=0$. So system (4) has no exponential factor. For $s>1$, set $g_{s-1}=z p_{s-2}(x, y, z)+q_{s-1}(x, y)$ with $p_{s-2}$ and $q_{s-1}$ homogeneous polynomials of degrees $s-2$ and $s-1$, respectively. Then we obtain from (9) with $z=0$ that $y \partial q_{s-1} / \partial y=-s q_{s-1}$. This last equation has only the solution $q_{s-1}(x, y)=0$. So we have $g_{s-1}=z p_{s-2}(x, y, z)$.

From equation (9) we get that

$$
y(y-2 z) \frac{\partial p_{s-2}}{\partial y}-(y-z)\left(p_{s-2}+z \frac{\partial p_{s-2}}{\partial z}\right)=-s(y-z) p_{s-2}+L_{0} z^{s-1} .
$$

If $s=2$, we get from (10) that $p_{s-2}=0$, and so system (4) has no exponential factor. For $s>2$, set $p_{s-2}=z p_{s-3}(x, y, z)+q_{s-2}(x, y)$ with $p_{s-3}$ and $q_{s-2}$ homogeneous polynomials of degrees $s-3$ and $s-2$, respectively. Then we obtain from (10) with $z=0$ that $y \partial q_{s-2} / \partial y=-(s-1) q_{s-2}$. This equation has only the solution $q_{s-2}(x, y)=0$. So we have $p_{s-2}=z p_{s-3}(x, y, z)$. Moreover $p_{s-3}$ satisfies the following equation

$$
y(y-2 z) \frac{\partial p_{s-3}}{\partial y}-(y-z)\left(2 p_{s-3}+z \frac{\partial p_{s-3}}{\partial z}\right)=-s(y-z) p_{s-3}+L_{0} z^{s-2} .
$$

This equation has a similar form than (10).

By induction we can prove that $g_{s-1}=b z^{s-1}$ with $b$ a constant. Substituting $g_{s-1}$ into equation (9) yields $b=0$. So $g$ has degree at most $s-2$.

If $\operatorname{deg} g=k \leq s-2$, then equation (5) implies that $L=0$. Moreover, we have

$$
y(y-2 z) \frac{\partial g_{k}}{\partial y}-z(y-z) \frac{\partial g_{k}}{\partial z}=-s(y-z) g_{k} .
$$

Working in a similar way to the proof of (9) we can prove that (11) has only the solution $g_{k}=0$.

The above proof shows that system (4) has no exponential factor associated with the invariant plane $z=0$. This completes the proof of the proposition. 


\section{ACKNOWLEDGMENTS}

We thank to the referee his comments which help us to improve the presentation of this paper.

The author is partially supported by the grants MICINN/FEDER MTM 200803437, AGAUR 2009SGR410 and ICREA Academia. The second author is partially supported by NNSF of China grant 10831003 and Shanghai Pujiang Programm 09PJD013.

\section{REFERENCES}

[1] A. Campillo and M.M. Carnicer, Proximity inequalities and bounds for the degree of invariant curves by foliations of $\mathbf{P}_{\mathbb{C}}^{2}$, Trans. Amer. Math. Soc. 349 (1997), 2211-2228.

[2] M.M. Carnicer, The Poincaré problem in the nondicritical case, Annals of Math. 140 (1994), 289-294.

[3] D. Cerveau and A. Lins Neto, Holomorphic foliations in CP(2) having an invariant algebraic curve, Ann. Inst. Fourier 41 (1991), 883-903.

[4] J. Chavarriga, H. Giacomini, J. Giné and J. Llibre, Darboux integrability and the inverse integrating factor, J. Differential Equations 194 (2003), 116-139.

[5] J. Chavarriga and M. Grau, A Family of non-Darboux integrable quadratic polynomial differential systems with algebraic solutions of arbitrarily high degree, Applied Math. Letters 16 (2003), 833-837.

[6] C.J. Christopher, Invariant algebraic curves and conditions for a center, Proc. Roy. Soc. Edinburgh 124A (1994), 1209-1229.

[7] C. Christopher, Liouvillian first integrals of second order polynomial differential equations, Electron. J. Differential Equations 1999, no. 49, 7 pp.

[8] C. Christopher And J. Llibre, Algebraic aspects of integrability for polynomial systems, Qualitative Theory of Dynamical Systems 1 (1999), 71-95.

[9] C. Christopher and J. Llibre, Integrability via invariant algebraic curves for planar polynomial differential systems, Annals of Differential Equations 16 (2000), 5-19.

[10] C. Christopher And J. Llibre, A family of quadratic polynomial differential systems with invariant algebraic curves of arbitrarily high degree without rational first integrals, Proc. Amer. Math. Soc. 130 (2002), 2025-2030.

[11] C. Christopher, J. Llibre and J.V. Pereira, Multiplicity of invariant algebraic curves in polynomial vector fields, Pacific J. Math. 229 (2007), 63-117.

[12] A. Cima, J. Llibre, Bounded polynomial systems, Trans. Amer. Math. Soc. 318 (1990), $557-579$.

[13] G. Darboux, Mémoire sur les équations différentielles algébriques du premier ordre et du premier degré (Mélanges), Bull. Sci. Math. 2ème série 2 (1878), 60-96; 123-144; 151-200.

[14] G. Darboux, De l'emploi des solutions particulières algébriques dans l'intégration des systèmes d'équations différentielles algébriques, C. R. Math. Acad. Sci. Paris 86 (1878), $1012-1014$.

[15] V.A. Dobrovol'skit, N.V. Lokot' And J.-M STrelcyn, Mikhail Nikolaevich Lagutinskii (1871-1915): un mathématicien méconnu, Historia Math. 25 (1998), 245-264.

[16] F. Dumortier, J. Llibre And J.C. Artés, Qualitative theory of planar differential systems, UniversiText, Springer-Verlag, New York, 2006.

[17] A. Ferragut, J. Llibre and A. Mahdi, Polynomial inverse integrating factors for polynomial vector fields, Discrete Contin. Dyn. Syst. 17 (2007), 387-395.

[18] J. Giné And J. Llibre, A family of isochronous foci with Darboux first integral, Pacific J. Math. 218 (2005), 343-355.

[19] D. Hilbert, Mathematische Probleme, Lecture, Second Internat. Congr. Math. (Paris, 1900), Nachr. Ges. Wiss. G"ttingen Math. Phys. KL. (1900), 253-297; English transl., Bull. Amer. Math. Soc. 8 (1902), 437-479.

[20] J.P. Jounnolou, Equations de Pfaff algébriques, in Lectures Notes in Mathematics 708, Springer-Verlag, New York/Berlin, 1979.

[21] A. Lins Neto, Some examples for the Poincaré and Painlevé problems, Ann. Sci. École Norm. Sup. 35 (2002), 231-266. 
[22] J. LLiBRe, Integrability of polynomial differential systems, in Handbook of differential equations, Elsevier, Amsterdam, 2004, pp.437-532.

[23] J. Llibre And J.C. Medrado, On the invariant hyperplanes for d-dimensional polynomial vector fields, J. Phys. A: Math. Gen. 40 (2007), 8385-8391.

[24] J. Llibre and G. Rodríguez, Configurations of limit cycles and planar polynomial vector fields, J. Diff. Eqns. 198 (2004), 374-380.

[25] J. Llibre and C. Valls, Integrability of the Bianchi IX system, J. Math. Phys. 46 (2005), 072901, 1-13.

[26] J. Llibre And C. VAlls, On the integrability of the Einstein-Yang-Mills equations, J. Math. Anal. Appl. 336 (2007), 1203-1230.

[27] J. Llibre AND X. ZhAnG, Invariant algebraic surfaces of the Lorenz systems, J. Mathematical Physics 43 (2002), 1622-1645.

[28] J. Llibre and X. Zhang, Darboux Theory of Integrability in $\mathbb{C}^{n}$ taking into account the multiplicity, J. of Differential Equations 246 (2009), 541-551.

[29] J. Llibre AND X. Zhang, Darboux theory of integrability for polynomial vector fields in $\mathbb{R}^{n}$ taking into account the multiplicity at infinity, Bull. Sci. Math. 133 (2009), 765-778.

[30] J. Llibre and X. Zhang, Rational first integrals in the Darboux theory of integrability in $\mathbb{C}^{n}$, Bull. Sci. Math. 134 (2010), 189-195.

[31] sc Yiu-Kwong Man and M.A.H. Maccallum, A Rational Approach to the Prelle-Singer Algorithm, J. of Symbolic Computation 24 (1997), 31-43.

[32] J. Moulin Ollagnier, About a conjecture on quadratic vector fields, Journal of Pure and Applied Algebra 165 (2001), 227-234.

[33] J.M. Pearson, N.G. Lloyd and C.J. Christopher, Algorithmic derivation of centre conditions, SIAM Review 38 (1996), 619-636.

[34] J.V. Pereira, Vector fields, invariant varieties and linear systems, Annales de l'institut Fourier 51 (2001), 1385-1405.

[35] J.V. Pereira, Integrabilidade de equaçoes diferenciais no plano complexo, Monografias del IMCA 25, Lima, Peru, 2002.

[36] H. Poincaré, Sur l'intégration des équations différentielles du premier ordre et du premier degré I and II, Rendiconti del Circolo Matematico di Palermo 5 (1891), 161-191; 11 (1897), 193-239.

[37] M.J. Prelle And M.F. Singer, Elementary first integrals of differential equations, Trans. Amer. Math. Soc. 279 (1983), 613-636.

[38] D. Schlomiuk, Algebraic particular integrals, integrability and the problem of the center, Trans. Amer. Math. Soc. 338 (1993), 799-841.

[39] D. Schlomiuk and N. Vulpe, Planar quadratic vector fields with invariant lines of total multiplicity at leat five, Qual. Theory Dyn. Syst. 5 (2004), 135-194.

[40] M.F. Singer, Liouvillian first integrals of differential equations, Trans. Amer. Math. Soc. 333 (1992), 673-688.

[41] C. VAlls, Rikitake system: analytic and Darbouxian integrals, Proc. Roy. Soc. Edinburgh Sect. A 135 (2005), 1309-1326.

[42] S. Walcher, On the Poincaré problem, J. Differential Equations 166 (2000), 51-78.

[43] X. Zhang, Exponential factors and Darbouxian first integrals of the Lorenz system, J. Math. Phys. 43 (2002), 4987-5001. 